\title{
A NEW CADMIUM-VAPOR ARC LAMP
}

\author{
By Frederick Bates
}

The necessity for increasing the intensity as well as the number of monochromatic-light sources has been emphasized frequently during the past few years. Unfortunately, little has been accomplished toward attaining this objective. In 1906 the writer ${ }^{1}$ directed attention to the importance of this subject and suggested that the so-called yellow-green line $(\lambda=546 \mathrm{I} A)$ of incandescent mércury vapor be adopted as the source for standardization purposes in polarimetric work. The quartz-mercury vapor lamp was a great advance in that it provided not only the yellow-green line, but several additional lines of lesser intensity. The best available methods of optical purification are such that a monochromatic source is of little value unless the line is so sufficiently removed from its immediate neighbors that nearly complete separation by spectrum filtration is possible. If sufficient monochromatic light to satisfy modern practical and research needs is to be obtained from any such source, it is necessary to use a relatively wide slit, with a consequent probable inclusion of other wave lengths in the immediate vicinity of the one desired.

When the most intense of all known light sources-namely, the direct radiation of the sun-is utilized, the necessary slit width, while less than that for any other known source, must still be such as to include a relatively large number of wave lengths. The effective wave length, or so-called optical center of gravity of such a group of waves, can be considered as a monochromaticlight source in only a very restricted sense and finds effective application in but few fields of work. It is especially unsuited to the study of phenomena which change rapidly with a change of wave length. The necessity for obtaining additional intenselight sources is consequently imperative.

Among the possible sources which have been suggested is that of the rotating arc with cadmium-silver alloy electrodes. This source gives a number of fairly intense lines sufficiently isolated 
from each other and fairly well distributed throughout the spectrum. The writer has carried out many experiments with this source, using an improved rotating arc. It was found impossible to maintain an arc sufficiently free from flicker to give satisfactory results.

Another possible source experimented with is the quartzcadmium vapor arc lamp, described by Lowry and Abram. ${ }^{2}$ This lamp is always unsatisfactory, owing to two defects. It is necessary to have it permanently connected to an air pump and to immerse the electrodes in water. If the cadmium in a vapor lamp is sufficiently pure, the adhesion between the cadmium and the quartz results in the destruction of the lamp upon the solidification of the cadmium. An improved form of lamp has been brought out by Sand. ${ }^{3}$ In this type the tendency of the cadmium to adhere to the quartz walls is stated to be lessened by introducing into the lamp a small amount of zirconia in the form of a fine powder. The cadmium is placed in a side tube connected to the pump and to the body of the lamp by a tube constricted to three capillaries for the purpose of filtering the metal. Additional filtering may be obtained by introducing a roll of iron gauze. Extensive experiments by the writer with this type of lamp have demonstrated that it is impracticable if a pure cadmium spectrum is desired. The method of filtering suggested is inadequate. The impurities introduced into the lamp by this method of filling undoubtedly have a tendency to prevent breakage, but effectively prevent obtaining a relatively pure, intense cadmium spectrum. In order to eliminate all oxide and other impurities from the cadmium used in filling, it is necessary to carefully distill the cadmium into the body of the lamp. Upon allowing the lamp to cool, adhesion between the quartz and the metal takes place in spite of the presence of the zirconia. If the lamp does not crack upon the first solidification of the cadmium, thin sections of the quartz are peeled from the walls by the contracting metal. Upon cooling a second time the lamp invariably cracks.

Numerous experiments of varied character failed to overcome the continued breakage of the Sand lamp. Among the filling mixtures tried was a cadmium-mercury alloy. The percentages of the constituents were varied over a wide range. The introduction of the mercury was very effective in preventing the crack-

2 Trans. Faraday Soc., 10, p. ro3; rgr4.

3 Proc. Phys. Soc., London, 28, p. 94; 1915-16. 
ing of the lamp, as the alloy formed was so soft that no appreciable adhesion between it and the quartz resulted. However, it was found impossible to obtain a brilliant cadmium spectrum under any circumstances. The vapor pressure of the mercury was so much higher than that of the cadmium that the electric energy was carried almost entirely by the mercury, and the usual brilliant mercury spectrum resulted.

In view of the preceding facts, it was evident that a serviceable, brilliant, cadmium-vapor lamp might be obtained by alloying the cadmium with a suitable element of lower vapor pressure. Through the courtesy of Dr. W. F. Hillebrand, a quantity of the little-known element gallium was obtained. The material was in a very impure condition, containing approximately ro per cent indium. The freezing point was below $22^{\circ} \mathrm{C}$, at which temperature it was a liquid with a viscosity less than that of mercury. A study of the impure material was made by Dr. G. E. F. Lundell, who succeeded in obtaining the gallium in a relatively pure condition.

Crude gallium was dissolved in aqua regia, treated with sulphuric acid, and fumed to remove nitric acid. After dilution, small amounts of lead sulphate were filtered off. The solution was then diluted, treated with hydrogen sulphide, and filtered to remove the hydrogen-sulphide group of elements. The filtrate was boiled to expel hydrogen sulphide and treated with ammonium hydroxide. The precipitate was filtered off, dissolved, and reprecipitated three times to free it from zinc. The final separation from indium was based on the solubility of gallium hydroxide in a solution of sodium hydroxide and the insolubility of indium hydroxide in that reagent. The sodium hydroxide separation was carried through three times. The deposition of gallium was finally carried out by electrolysis of the alkaline solution, as recommended by Uhler and Browning. ${ }^{4}$

The purified gallium had a freezing point of approximately $30^{\circ} \mathrm{C}$. This surprising fact has since been verified by the careful work of Richards ${ }^{5}$ who has definitely fixed this temperature at $30.8^{\circ} \mathrm{C}$. Little is known regarding the boiling point of this element. The few experiments which have been made are in agreement that it is above $1500^{\circ} \mathrm{C}$. This property should make gallium an ideal substance for the purpose in hand, provided it would alloy with cadmium. The first experiment demonstrated 
that it unites with cadmium with the utmost ease. In fact, the addition of a few drops to Io or I 5 cc of cadmium completely changed the texture of the latter, rendering it relatively soft and greatly reducing its tensile strength. Subsequently it was discovered that upon distilling the cadmium from the alloy at a pressure of $0.001 \mathrm{~mm}$ of mercury, the minute quantity of gallium carried through was sufficient to change completely the character

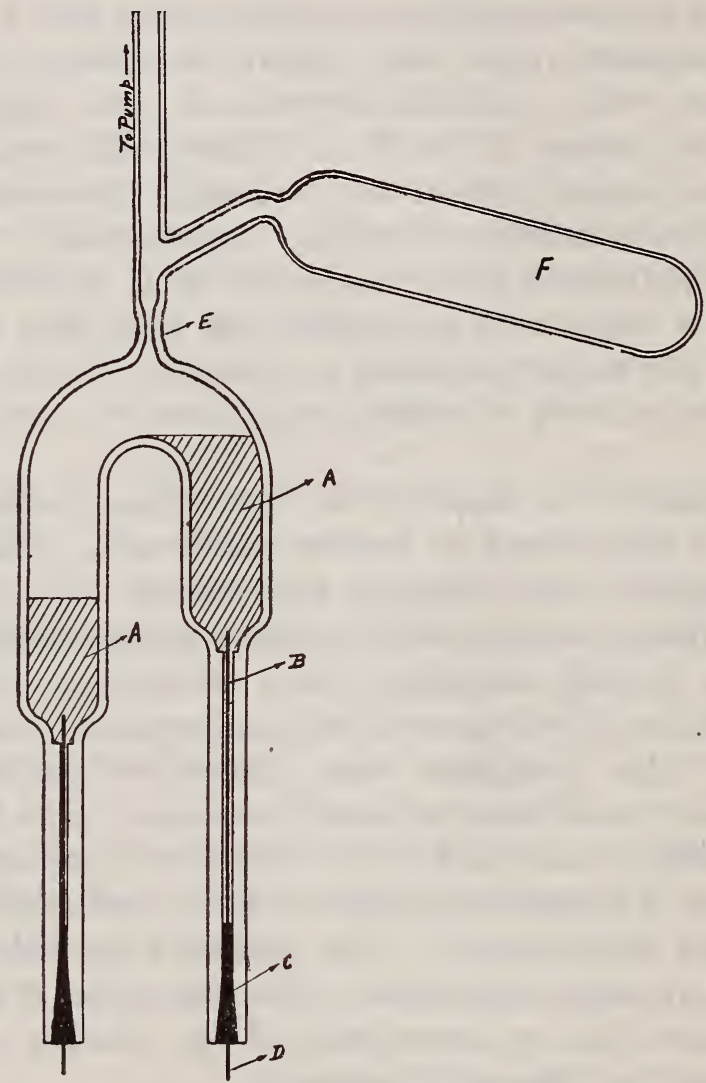

FIG. I.-Cross section of cadmium lamp with tube for distilling amalgam into the lamp

of the cadmium and to prevent adhesion between the cadmium and the walls of the lamp.

The type of quartz lamp used in the experiments is that shown in Fig. I. The total volume is approximately Io cc. The electrodes consist of tungsten wires $B$ entering through quartz capillaries. These are closed with lead seals similar to the type described by Sand. ${ }^{6}$ 
In filling the lamp, the cadmium, containing 2 or 3 per cent gallium, is placed in the bulb $F$. It is necessary to maintain the pressure in the lamp and connections below $0.001 \mathrm{~mm}$ of mercury, with the exception of that due to the cadmium and gallium, throughout the process of distilling. Since the volume of the lamp is relatively small, the quartz capillary at $E$ should be of such a length as to permit of sealing off in the shortest possible time. The flame used for this purpose should be small and the heating of the tube on both sides of the capillary prevented as far as possible.

The method indicated above, if carefully followed, will yield a lamp with indefinite life. One of this type has been in intermittent use for over a year and shows no sign of deterioration. Should traces of oxide or stains due thereto appear during the process of filling, they can be reduced readily by introducing pure, dry hydrogen and heating. The lamp may be started by heating with a flame to vaporize the metal. It is in all cases advisable to

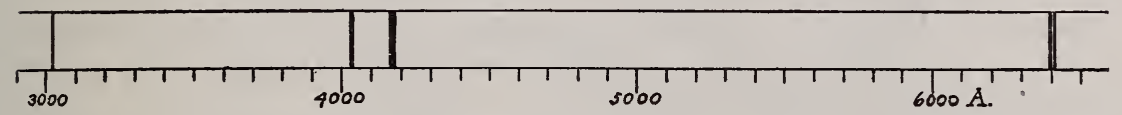

FIG. 2.-Gallium spectrum

have a current of air blowing upon the lead seals to keep them cool. If the blast is allowed to strike the body of the lamp, the cadmium is condensed and obscures the arc. The most convenient source of energy for operation is the ordinary r ro-volt lighting circuit, on which it will operate continuously with a current as small as 3 amperes and a drop of 14 volts across the terminals of the lamp. However, the most satisfactory results are secured with a current of about 7 amperes and a drop across the terminals of about 25 volts. Under this condition a practically pure cadmium spectrum of great brilliancy is obtained. The intensity secured is apparently equal to that which would be obtained were the lamp filled with cadmium alone. The map ${ }^{7}$ of the spectrum of gallium given in Fig. 2 is interesting. In this connection the wave lengths and intensities of the lines are given in Table I. It will be observed that there are but four lines in the visible spectrum, and that from practically $4200 \mathrm{~A}$ to $6400 \mathrm{~A}$ there are no lines. 
TABLE 1.-Visible Spectrum of Gallium

\begin{tabular}{|c|c|}
\hline Wave length A & Intensity \\
\hline 3020.61 & 3 \\
4033.18 & 10 \\
4172.22 & 20 \\
6396.99 & 8 \\
6413.92 & 6 \\
\hline
\end{tabular}

When the lamp is operated at a temperature sufficiently high to bring the quartz to a cherry-red color and there is danger of softening the lamp, several gallium lines become faintly visible. The investigations of Uhler and Browning ${ }^{8}$ indicate the possibility of two gallium lines 5353.8 I A and 5359.8 A. However, these lines, if present, are so faint at the highest temperature at which the lamp can be operated that they can not be identified. The cadmium spectrum is thus obtained in a condition exceedingly favorable for those purposes for which an intense monochromatic light source is indispensable. No gallium lines are found between $4200 \mathrm{~A}$ and $6400 \mathrm{~A}$, and the gallium lines which are detectable have so low an intensity that they are wholly negligible in polarimetric and other fields of work.

To summarize, there are now available practically no dependable intense monochromatic red-light sources. Any source to meet modern demands must permit of continuous operation with a minimum amount of attention and an absence of flicker. The very pure red line $(\lambda=6439 \mathrm{~A})$ of cadmium seems to be the only possible source of sufficient intensity in this region of the spectrum. It is believed that the cadmium-gallium lamp will make this muchneeded source, as well as other lines of the cadmium spectrum, available for many lines of endeavor.

The writer desires to acknowledge his indebtedness to F. P. Phelps for valuable assistance in the experimental work.

Washington, December I, I9I9. 